\title{
Factores que Afectan la Adopción de Tecnologías de Información en Micro y Pequeñas empresas: un Estudio Cualitativo
}

\author{
María de León-Sigg ${ }^{1}$, Sodel Vázquez-Reyes ${ }^{2}$, Juan L. Villa-Cisneros ${ }^{3}$ \\ mleonsigg@uaz.edu.mx, vazquezs@uaz.edu.mx,jlvilla@uaz.edu.mx \\ 123 Profesor Investigador de la UAZ, Edificio de Ingeniería en Computación y Software, 1er Piso. Carretera a \\ Guadalajara Km 6, Ejido La Escondida, 98160, Zacatecas, México.
}

DOI: 10.17013/risti.22.20-36

\begin{abstract}
Resumen: Las micro y pequeñas empresas son la principal fuente de empleos en toda América Latina y México. Por ello, buscar formas para consolidarlas es un área importante de interés. Sin embargo, poca investigación se ha hecho para encontrar factores que afectan la adopción de tecnologías de la información en áreas de bajo crecimiento económico. Con esto en mente, se realizó una investigación cualitativa que encontrara factores internos y externos que afectan la adopción en micro y pequeñas empresas ubicadas en la zona metropolitana de Zacatecas, México. Los resultados revelan que los factores internos coinciden con aquellos encontrados en la revisión de la literatura, pero los externos no. Estos resultados podrían utilizarse para iniciar nuevas investigaciones y modificar creencias existentes sobre el entrenamiento y la consolidación de micro y pequeñas empresas a través de la adopción de tecnologías de la información.
\end{abstract}

Palabras-clave: Tecnología de Información, adopción, micro y pequeñas empresas, estudio cualitativo.

Factors affecting Information Technology adoption in micro and small enterprises: a qualitative study

\begin{abstract}
Micro and small enterprises are the main source of jobs across Latin America and Mexico. Thus, searching ways to consolidate them is a major area of interest. However, poorly research has been done to find factors affecting Information Technology adoption in low economic growth areas. With this in mind, a qualitative research was done to find internal and external factors that affect adoption in micro and small enterprises in the metropolitan area of Zacatecas, Mexico. Results demonstrate that internal factors coincide with literature review, but external ones not. These results could be used to initiate new research and to modify existent beliefs about training and consolidating micro and small enterprises.
\end{abstract}

Keywords: Information technology, adoption, micro and small enterprises, qualitative study. 


\section{Introducción}

Debido a la importancia económica de las micro y pequeñas empresas, se han buscado medios que apuntalen su desarrollo. Uno de estos medios es la adopción y uso de Tecnologías de Información (TI), ya que permiten que las organizaciones tengan información valiosa a su alcance, aumenten su conocimiento, mejoren las relaciones con los clientes y proveedores, profundicen y mantengan la colaboración con otras compañías, aumenten su eficiencia, ofrezcan nuevos canales de comunicación y distribución, y reduzcan costos de producción (Maldonado-Guzmán, Martínez-Serna, García Perez de Lema, Aguilera Enríquez, \& González Adame, 2010; Parida, Johansson, Ylinenpää, \& Baunerhjelm, 2010; Qureshi, Kamal, \& Wolcott, 2009; Sá \& Rocha, 2012). Sin embargo, en México y Latinoamérica, la investigación sobre la adopción de TI se ha dirigido principalmente en el tipo y cantidad de TI en uso o en las consecuencias del uso de las mismas (AMIPCI, 2010; Aregional, 2010; Arellano-González, Carballo-Mendivil, Orrantia-López, \& SalazarRivera, 2013; Biasca, 2010; Maldonado-Guzmán et al., 2010; Rubio Bañón \& Aragón Sánchez, 2002), pero no en los factores que determinaron su adopción.

Por lo anterior, el propósito de la investigación aquí presentada, fue examinar los factores que afectan la adopción de TI en micro y pequeñas empresas, en el contexto de un área geográfica como la zona metropolitana de Zacatecas (Trejo Nieto, 2013). En la siguiente sección se expone la literatura revisada sobre el tema. La sección tres describe la metodología utilizada para la investigación. En la sección cuatro se presentan y discuten los resultados obtenidos, y, finalmente, en la sección cinco se encuentran las conclusiones y los trabajos futuros derivados de la investigación.

\section{Revisión de literatura}

Los factores que mayor influencia tienen sobre la adopción de TI en micro y pequeñas empresas han sido clasificados como internos y externos (Drew, 2003; Ghobakhloo, Hong, Sabouri, \& Zulkifli, 2012). Los factores se clasifican como internos, si están dentro del contexto organizacional y tecnológico de la empresa, y, por lo tanto, son controlables; y externos, si se encuentran fuera de éste, y por lo tanto son menos controlables o totalmente incontrolables para la organización. Tomando como referencia la clasificación de factores de Ghobakhloo et. al (2012), y el marco teórico de Ramaya et. al (Ramayah, Ling, Taghizadeh, \& Rahman, 2015), se encontró que los factores internos que impactan la adopción de TI son el administrador y sus características, los recursos con los que cuenta la empresa y, por último, los usuarios de estas tecnologías, entre los que se encuentran los empleados de las empresas. Por el lado de los factores externos están el gobierno, en forma de las políticas y regulaciones que afectan a las micro y pequeñas empresas, los clientes, la competencia y los vendedores y consultores de TI. En la Tabla 1 se resume la literatura revisada para esta investigación. La búsqueda de referentes se hizo en Elsevier, Springer, Wiley y Ebsco, considerando como criterios de búsqueda “micro y pequeñas empresas”, “adopción”, “tecnología de información”, en inglés y en español. Se consideraron solamente estudios de empresas que concuerdan con la definición de micro y pequeña empresa de acuerdo con la clasificación oficial mexicana (Secretaría de Gobernación, 2009). Además, la búsqueda se limitó a trabajos publicados del año 2000 a 2015. 


\begin{tabular}{lll}
\hline Criterio & Fuente & $\begin{array}{l}\text { Fuente de México o América } \\
\text { Latina }\end{array}$ \\
\hline & Factores Internos \\
\hline Administrador & &
\end{tabular}

Percepción hacia la

Tecnología de Información

(costos, beneficios, control)

Percepción hacia la

alineación con la estrategia

de la Tecnología de

Información
(Cragg, King, \& Hussin, 2002), (Fink \& Disterer, 2006), (Qureshil \& York, 2008), (Ramdani, Kawalek, \& Lorenzo, 2007) 2009)

(Utomo \& Dodgson, 2001)

(Seyal, Rahim, \& Rahman, 200o), (Utomo \& Dodgson, 2001), (Ihlström, Magnusson, Scupola, \& Tuunainen, 2003), (Ward \& Caldeira, 2003), (Chao \& Chandra, 2012)
(Arroyo, Ramírez, \& Erosa-Martín,

(Middleton \& Byus, 2011), (Jones, Motta, \& Alderete, 2016)

(Ueki, Tsuji, \& Cárcamo-Olmos, 2005), (Erosa-Martín \& ArroyoLópez, 2010), (Brewerton \& Feinberg, 2011), (Middleton \& Byus, 2011) (González-Peyro, Torres-Nájera, \& SifuentesGarcía, 2012), (de León-Sigg, Villa-Cisneros, Vazquez-Reyes, \& Rentería-Salcedo, 2013)

(Riemenschneider, Harrison, \&

(Amorós, Planellas, \& BatistaFoguet, 2007) Mykytyn, 2003), (Qureshil \& York, 2008)

Actitud hacia la Tecnología de Información

Liderazgo del administrador (Spencer, Buhalls, \& Moital, 2012)

\section{Usuarios}

Conocimiento y habilidades de los usuarios

Actitud y opinión de los usuarios
(Thong, 2001), (Bruque \& Moyano, 2007), (Alam \& Noor, 2009)

(Love, Irani, Standing, Lin, \& Burn, 2005)
(Ueki et al., 2005), (Siqueira \&

Fleury, 2011), (Novaes-Zilber \& Braz-de Araújo, 2012)

(Antonelli, Brito-de Almeida, Bortolocci-Espejo, \& Longhi, 2013)

\section{Recursos}

Recursos financieros e inversión
(Thong, 2001), (Ihlström et al., 2003), (Sarosa \& Zowghi, 2003), (Mole \& Ghobadian, 2004), (Tan, Chong, Lin, \& Eze, 2009), (Nguyen, 2009), (Benitez-Amado, Llorens-Montes, \& Perez-Arostegui, 2010)
(Ueki et al., 2005)

\section{Factores externos}

\section{Clientes}

(Ihlström et al., 2003),

(Riemenschneider et al., 2003),

Presión de clientes

(Ward \& Caldeira, 2003), (Mole \& Ghobadian, 2004), (Búrca, Fynes, \& Marshall, 2005), (Nguyen, Newby, \& (Guerrero-Cuéllar \& Rivas-Tovar, 2005)

Macaulay, 2013)

\section{Gobierno}


(Utomo \& Dodgson, 2001), (Sarosa

Apoyo y políticas

gubernamentales
\& Zowghi, 2003), (Fathian, Akhavan,

\& Hoorali, 2008), (Tan et al., 2009),

(Alam \& Noor, 2009)
(Kuwayama, 2001), (Botelho

\& Bastos-Tigre, 2005), (Erosa-

Martín \& Arroyo-López, 2010),

(Merritt, 2012)

Vendedores y consultores

(Walczuch, Van Braven, \& Lundgren, 2000), (Thong, 2001), (Stockdale \&

Apoyo y servicio de los Standing, 2004) (Salmeron \& Bueno, 2006), (Nguyen, 2009), (Ghobakhloo, Arias-Aranda, \& Benitez-Amado, 2011)

Competencia

Actitud de la competencia hacia la Tecnología de Información

(Nguyen, 2009), (Premkumar, 2003), (Riemenschneider et al., 2003), (Drew, (Guerrero-Cuéllar \& Rivas-Tovar, 2003), (Mole \& Ghobadian, 2004), 2005)

(Salmeron \& Bueno, 2006)

(Sarosa \& Zowghi, 2003), (Pontikakis,

Presión por competir Lin, \& Dermibas, 2006), (Ghobakhloo et al., 2011).

Tabla 1 - Factores que afectan la adopción de Tecnologías de Información en micro y pequeñas empresas

Es importante hacer notar que el administrador juega un papel determinante en la adopción de TI, puesto que en las micro y pequeñas empresas es el principal, y en ocasiones único, tomador de decisiones (Chesney, 2010).

\section{Metodología}

En esta investigación se utilizó un enfoque cualitativo, para comprender la perspectiva de los administradores de micro y pequeñas empresas localizadas en el área metropolitana de Zacatecas, acerca de la adopción de TI. Este enfoque ha sido utilizado con anterioridad para la investigación relacionada con el área de conocimiento de TI (Myers \& Baskerville, 2004; Walsham, 2006; Yen \& Bakken, 2012). Además, se utilizó el estudio de casos para tener un mejor entendimiento y confirmación del marco de investigación teórico (Jabar, Sidi, Selamat, Ghani, \& Ibrahim, 2009), expuesto en la sección anterior. Para esta investigación se consideró una muestra teórica de seis empresas que fueron elegidas de acuerdo con los siguientes criterios:

- Tamaño. Se eligieron micro y pequeñas empresas, puesto que en la zona metropolitana de Zacatecas, son las que se presentan con mayor frecuencia (INEGI, 2013).

- Ubicación. Se eligieron empresas ubicadas en la zona metropolitana de Zacatecas, puesto que es la más importante del Estado de Zacatecas (Secretaría de Desarrollo Social, Consejo Nacional de Población, \& INEGI, 2004).

- Sector. Se eligieron empresas de los sectores de servicios y comercio debido a que son el tipo de empresas que tienen una aportación mayor al PIB estatal (INEGI, 2013). 


\begin{tabular}{lcc}
\hline Categoría & Subcategorías & Esquema de codificación y asignación de valor \\
\hline & Factores Internos \\
\hline
\end{tabular}

Percepción sobre TI Efectividad, beneficio, $\quad$ Negativa (N): el administrador no percibe el aspecto relación costo/beneficio, listado como resultado de la adopción de TI en su control empresa.

Positiva y Negativa $(\mathrm{P}+\mathrm{N})$ : el administrador tiene percepciones encontradas sobre el aporte de las TI en el aspecto listado.

Positiva (P): el administrador percibe el aspecto listado como resultado de la adopción de TI en su empresa.

Percepción hacia la alineación con la estrategia
Visión del futuro de la empresa, Uso de las TI para cumplir los objetivos de negocio de la empresa
Inexistente (I): el administrador no identifica con claridad el futuro posible de su empresa.

Relativamente definida (R): el administrador identifica con alguna claridad el futuro posible de su empresa.

Definida (D): el administrador tiene una idea clara del futuro que quiere para su empresa.

Experiencia y conocimiento de TI

Nivel de educación, nivel de conocimiento

Bajo (B): el administrador no tiene cursos o talleres de formación en TI.
Medio (M): el administrador ha hecho cursos o talleres llevados a cabo durante la educación formal. Alto (A): el administrador ha hecho cursos o talleres obtenidos fuera de la educación formal (educación continua).

\begin{tabular}{ll}
\hline Actitudes hacia las & $\begin{array}{l}\text { Predisposición hacia las TI, } \\
\text { Apoyo a las iniciativas de } \\
\text { adopción }\end{array}$
\end{tabular}

Negativa (N): el administrador considera que las TI son difíciles de utilizar y complican su trabajo. Positiva y Negativa $(\mathrm{P}+\mathrm{N})$ : el administrador considera un "mal necesario" a las TI, al menos en algunos casos.

Positiva (P): el administrador considera que las TI le permiten ser más productivo y eficiente en sus tareas.

\begin{tabular}{lll}
\hline Liderazgo & $\begin{array}{l}\text { Principal tomador de } \\
\text { decisiones con respecto a } \\
\text { la adopción de TI, Criterios } \\
\text { tomados en cuenta para } \\
\text { la toma de decisiones con } \\
\text { respecto a la adopción de TI }\end{array}$ & $\begin{array}{l}\text { Bajo (B): el administrador nunca o casi nunca toma } \\
\text { decisiones sobre adopción de TI. } \\
\text { Medio (M): el administrador toma decisiones junto } \\
\text { con alguien más y su nivel de toma de decisiones es } \\
\text { mediano sobre adopción de TI. } \\
\text { Alto (A): el administrador es el principal tomador de } \\
\text { decisiones sobre la adopción de TI. }\end{array}$ \\
& Nivel de educación, Nivel de & $\begin{array}{l}\text { Bajo (B): el usuario tiene educación básica (primaria, } \\
\text { secundaria), sin cursos o talleres de formación en TI. } \\
\text { conocimiento }\end{array}$ \\
$\begin{array}{ll}\text { Medio (M): el usuario tiene educación superior } \\
\text { (preparatoria, licenciatura), con cursos o talleres } \\
\text { habilidades de los } \\
\text { usuarios }\end{array}$ & $\begin{array}{l}\text { llevados a cabo durante la educación formal. } \\
\text { Alto (A): el usuario tiene posgrado, con cursos o } \\
\text { talleres obtenidos fuera de la educación formal } \\
\text { (educación continua). }\end{array}$
\end{tabular}




\begin{tabular}{|c|c|c|}
\hline $\begin{array}{l}\text { Actitud y opinión de } \\
\text { los usuarios }\end{array}$ & $\begin{array}{l}\text { Predisposición hacia las TI, } \\
\text { Apoyo a las iniciativas de } \\
\text { adopción }\end{array}$ & $\begin{array}{l}\text { Negativa (N): el usuario considera que las TI son } \\
\text { difíciles de utilizar y complican su trabajo. } \\
\text { Positiva y Negativa }(\mathrm{P}+\mathrm{N}) \text { : el usuario considera un } \\
\text { "mal necesario" a las TI, al menos en algunos casos. } \\
\text { Positiva (P): el usuario considera que las TI le } \\
\text { permiten ser más productivo y eficiente en sus tareas. }\end{array}$ \\
\hline $\begin{array}{l}\text { Recursos financieros } \\
\text { e inversión }\end{array}$ & $\begin{array}{l}\text { Asignación de presupuesto } \\
\text { para TI }\end{array}$ & $\begin{array}{l}\text { Si (S): la empresa cuenta con un presupuesto para } \\
\text { TI. } \\
\text { No (N): la empresa no cuenta con un presupuesto } \\
\text { para TI. } \\
\text { Si (S): la empresa cuenta con un presupuesto para TI. } \\
\text { No (N): la empresa no cuenta con un presupuesto } \\
\text { para TI }\end{array}$ \\
\hline
\end{tabular}

\section{Factores externos}

$\begin{array}{lll}\begin{array}{l}\text { Presión de los } \\ \text { clientes }\end{array} & \begin{array}{l}\text { Presión de los clientes } \\ \text { para la adopción de una }\end{array} & \begin{array}{l}\text { Bajo (B): el administrador no percibe la presión de } \\ \text { los clientes. }\end{array} \\ & \text { Tecnología o Sistema en } & \text { Medio (M): el administrador en ocasiones percibe } \\ & \text { particular, } & \text { esta presión y en ocasiones no es percibida. } \\ & \text { Presión por mejorar el } & \text { Alto (A): el administrador siente fuerte presión de } \\ & \text { servicio al cliente, Presión } & \text { parte de sus clientes en el aspecto indicado. } \\ & \text { por mejorar la presencia de } & \end{array}$
por mejorar la presencia de la empresa en el mercado

\begin{tabular}{ll}
\hline Apoyo y políticas & Políticas gubernamentales, \\
gubernamentales & Apoyos
\end{tabular}

Bajo (B): el administrador no considera las políticas gubernamentales o los apoyos como importante para la adopción de TI

Medio (M): el administrador considera en ocasiones las políticas gubernamentales o los apoyos como importante para la adopción de TI

Alto (A): el administrador considera muy importante las políticas gubernamentales o los apoyos para la adopción de TI.

\begin{tabular}{ll}
\hline Apoyo y servicio de & Apoyo y servicio post- \\
los vendedores & venta de los vendedores de \\
& TI, Tipo de TI vendidas, \\
& Adaptación a procesos
\end{tabular}

Bajo (B): el administrador indica que prácticamente no existe el apoyo y servicio de los vendedores. Medio (M): el administrador indica que en ocasiones existe el apoyo y servicio de los vendedores. Alto (A): el administrador indica que siempre existe el apoyo y servicio de los vendedores.

\begin{tabular}{ll}
\hline Actitud de la & Predisposición de la \\
competencia hacia & competencia hacia las TI, \\
las TI & Uso de TI
\end{tabular}
Negativa (N): el administrador percibe una actitud negativa de sus competidores hacia el uso de TI. Positiva y Negativa $(\mathrm{P}+\mathrm{N})$ : el administrador percibe una actitud regular de sus competidores hacia el uso de TI.

Positiva (P): el administrador percibe una fuerte actitud de sus competidores hacia el uso de TI.

\begin{tabular}{lll}
\hline $\begin{array}{l}\text { Presión por } \\
\text { competir }\end{array}$ & $\begin{array}{l}\text { Adopción de TI para } \\
\text { conseguir ventaja }\end{array}$ & $\begin{array}{l}\text { Bajo (B): el administrador no adopta TI para } \\
\text { competir. }\end{array}$ \\
& $\begin{array}{l}\text { competitiva, Presión por } \\
\text { utilizar TI como lo hace la } \\
\text { competencia }\end{array}$ & $\begin{array}{l}\text { Medio (M): el administrador considera como opción } \\
\text { la adopción TI para competir. }\end{array}$ \\
& $\begin{array}{l}\text { Alto (A): el administrador busca la adopción de TI } \\
\text { para mantenerse en el mercado. }\end{array}$ \\
\hline
\end{tabular}

El código "NA" se considera cuando el código no aplica por las condiciones de la empresa

Tabla 2 - Codificación 
- Consolidación. Las empresas elegidas para participar tienen más de dos años de fundadas.

- $\quad$ Propiedad. Los propietarios, fundadores y administradores de las empresas son zacatecanos.

- Relación con los investigadores. Se eligieron empresas con las que los investigadores no han tenido nunca relación laboral.

Para reunir la información se hicieron entrevistas semi estructuradas, basadas en

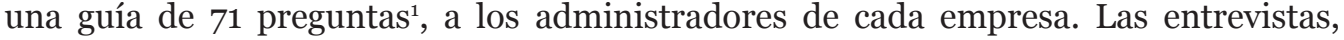
estructuradas o semi estructuradas, permiten que el investigador comprenda la situación en el contexto en el que está siendo estudiada, estudiar el comportamiento de los participantes en su entorno local y percibir lo que los participantes ven como relevante e importante (Babbie, 2009). Las entrevistas semi estructuradas, se basan en una guía de preguntas y el entrevistador tiene la libertad de introducir preguntas adicionales para precisar conceptos u obtener mayor información sobre los temas deseados (Hernández Sampieri, 2010). Las entrevistas fueron grabadas y transcritas.

Finalmente, se hizo un análisis de contenido a la transcripción, codificando la información recuperada de ellas (Neuendorf, 2001). El análisis de contenido ha sido previamente utilizado para estudiar la adopción de las TI en diferentes contextos (O’Mahony, Wright, Yogeswaran, \& Govere, 2014; Rese, Schreiber, \& Baier, 2014). El proceso de codificación implica la agrupación de información obtenida en categorías que concentran las ideas, conceptos o temas similares descubiertos por el investigador, a partir de una lista inicial de códigos. Para esta investigación, la lista inicial de códigos provino de los criterios mostrados en la Tabla 1. Dentro de cada categoría, se identificaron subcategorías en las que se identificaron las respuestas a las preguntas de las entrevistas, como se muestra en la Tabla 2.

\begin{tabular}{lllll}
\hline Empresa & $\begin{array}{l}\text { No. de } \\
\text { empleados } \\
\text { permanentes }\end{array}$ & $\begin{array}{l}\text { Año de } \\
\text { fundación }\end{array}$ & $\begin{array}{l}\text { Clasificación } \\
\text { (Micro } \\
\text { Pequeña) }\end{array}$ & Actividad económica \\
\hline 4 & 2010 & Micro & $\begin{array}{l}\text { Venta al por menor de muebles y } \\
\text { elementos de decoración }\end{array}$ \\
\hline 2 & 13 & 1983 & Pequeña & $\begin{array}{l}\text { Venta al por menor de perfumería } \\
\text { y regalo }\end{array}$ \\
\hline 3 & 8 & 2003 & Micro & Servicio de patología \\
\hline 4 & 2002 & Micro & $\begin{array}{l}\text { Venta al por menor de disfraces y } \\
\text { trajes típicos }\end{array}$ \\
\hline 5 & 25 & 2006 & Pequeña & Venta de pasteles al por menor \\
\hline 5 & 2004 & Micro & Servicio automotriz \\
\hline
\end{tabular}

Tabla 3 - Características de las micro y pequeñas empresas seleccionadas para la investigación

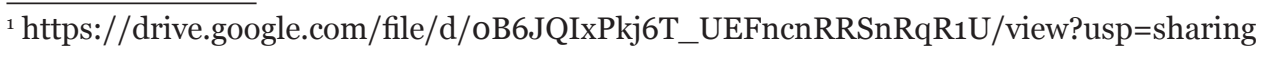




\section{Resultados}

En la Tabla 3 se muestra un resumen de las características de las empresas seleccionadas para el estudio, y que cumplen con lo indicado en la Metodología descrita antes.

En la Tabla 4, se resume la infraestructura de TI con la que cuentan las empresas revisadas. Sus edades variaron entre los 28 y los 63 años de edad. Todos ellos cuentan con estudios de nivel licenciatura y cuatro de ellos tienen maestría terminada o cursos de especialidad en administración de sistemas de cómputo.

\begin{tabular}{|c|c|c|c|c|c|}
\hline Empresa & $\begin{array}{l}\text { No. de } \\
\text { Compu- } \\
\text { tadoras }\end{array}$ & $\begin{array}{l}\text { Aplicaciones } \\
\text { comerciales }\end{array}$ & $\begin{array}{l}\text { Uso de } \\
\text { correo } \\
\text { electrónico/ } \\
\text { página web }\end{array}$ & $\begin{array}{l}\text { Procesos y } \\
\text { actividades de } \\
\text { negocio apoyados } \\
\text { por } \mathrm{TI}\end{array}$ & $\begin{array}{l}\text { Uso de } \\
\text { aplicaciones a } \\
\text { la medida }\end{array}$ \\
\hline 1 & 2 & $\begin{array}{l}\text { Compaq, } \\
\text { CorelDraw, } \\
\text { Office, Autocad }\end{array}$ & $\begin{array}{l}\mathrm{Si} / \mathrm{Si} \\
\text { (Facebook) }\end{array}$ & $\begin{array}{l}\text { Mostrar productos a } \\
\text { clientes, generar listas } \\
\text { de precios, enviar } \\
\text { cotizaciones, contactar } \\
\text { proveedores, generar } \\
\text { facturas electrónicas }\end{array}$ & $\begin{array}{l}\text { No. Uso de } \\
\text { bases de datos } \\
\text { en Excel }\end{array}$ \\
\hline 2 & $>2$ & Compaq, Office & $\mathrm{Si} / \mathrm{No}$ & $\begin{array}{l}\text { Registrar ventas y } \\
\text { compras, controlar } \\
\text { inventarios, realizar } \\
\text { análisis financiero, } \\
\text { contabilidad } \\
\text { electrónica }\end{array}$ & $\begin{array}{l}\text { No. Uso de } \\
\text { Excel para } \\
\text { proyecciones } \\
\text { financieras }\end{array}$ \\
\hline 3 & $>2$ & Office, Publisher & $\mathrm{Si} / \mathrm{No}$ & $\begin{array}{l}\text { Registrar servicios } \\
\text { ofrecidos, contactar } \\
\text { proveedores, } \\
\text { facturación electrónica }\end{array}$ & $\begin{array}{l}\text { Si. Historial de } \\
\text { servicios }\end{array}$ \\
\hline 4 & 2 & Office & $\mathrm{Si} / \mathrm{No}$ & $\begin{array}{l}\text { Controlar inventarios, } \\
\text { pagar impuestos, } \\
\text { controlar entradas y } \\
\text { salidas de empleados }\end{array}$ & $\begin{array}{l}\text { Si. Control de } \\
\text { asistencias y } \\
\text { puntualidad }\end{array}$ \\
\hline 5 & $>2$ & Office & $\begin{array}{l}\text { Si (Correo de } \\
\text { la empresa) } \\
\text { /Si }\end{array}$ & $\begin{array}{l}\text { Calendarización de } \\
\text { pedidos, manejo de } \\
\text { entradas y salidas de } \\
\text { dinero, control de } \\
\text { inventarios }\end{array}$ & $\begin{array}{l}\text { Si. Control de } \\
\text { producción }\end{array}$ \\
\hline 6 & 2 & $\begin{array}{l}\text { Office, } \\
\text { facturación } \\
\text { electrónica }\end{array}$ & $\begin{array}{l}\mathrm{Si} / \mathrm{Si} \text { (página } \\
\text { en Facebook) }\end{array}$ & $\begin{array}{l}\text { Registrar datos de } \\
\text { clientes, registrar } \\
\text { facturas, facturación } \\
\text { electrónica }\end{array}$ & $\begin{array}{l}\text { No. Uso de } \\
\text { bases de datos } \\
\text { en Excel }\end{array}$ \\
\hline
\end{tabular}

Tabla 4 - Infraestructura de TI de las empresas estudiadas

Como se puede observar, en la Tabla 5, las empresas estudiadas cuentan todas con equipo de cómputo y acceso a Internet, es decir con la infraestructura básica de TI.

En la Tabla 5 se presenta el resultado de la codificación de las transcripciones de las entrevistas de acuerdo a lo establecido en la Metodología. 


\section{Administradores entrevistados}

\begin{tabular}{lllllll}
\hline Categoría & $\mathbf{1}$ & $\mathbf{2}$ & $\mathbf{3}$ & $\mathbf{4}$ & $\mathbf{5}$ & $\mathbf{6}$ \\
\hline & Factores internos & & & & \\
\hline Percepción sobre TI & $\mathrm{P}$ & $\mathrm{P}$ & $\mathrm{P}$ & $\mathrm{P}$ & $\mathrm{P}$ & $\mathrm{P}$ \\
\hline $\begin{array}{l}\text { Percepción hacia la alineación con la } \\
\text { estrategia }\end{array}$ & $\mathrm{D}$ & $\mathrm{R}$ & $\mathrm{R}$ & $\mathrm{D}$ & $\mathrm{D}$ & $\mathrm{D}$ \\
\hline Experiencia y conocimiento de TI & $\mathrm{A}$ & $\mathrm{A}$ & $\mathrm{B}$ & $\mathrm{A}$ & $\mathrm{M}$ & $\mathrm{M}$ \\
\hline Actitudes hacia las TI & $\mathrm{P}+\mathrm{N}$ & $\mathrm{P}+\mathrm{N}$ & $\mathrm{P}$ & $\mathrm{P}+\mathrm{N}$ & $\mathrm{P}$ & $\mathrm{P}$ \\
\hline Liderazgo & $\mathrm{M}$ & $\mathrm{A}$ & $\mathrm{B}$ & $\mathrm{A}$ & $\mathrm{A}$ & $\mathrm{A}$ \\
\hline Conocimientos y habilidades de los usuarios & $\mathrm{M}$ & $\mathrm{M}$ & $\mathrm{B}$ & $\mathrm{M}$ & $\mathrm{M}$ & $\mathrm{NA}$ \\
\hline Actitud y opinión de los usuarios & $\mathrm{P}$ & $\mathrm{P}$ & $\mathrm{P}+\mathrm{N}$ & $\mathrm{P}$ & $\mathrm{P}$ & $\mathrm{NA}$ \\
\hline Recursos financieros e inversión & $\mathrm{N}$ & $\mathrm{N}$ & $\mathrm{N}$ & $\mathrm{N}$ & $\mathrm{N}$ & $\mathrm{N}$ \\
\hline Presión de los clientes & $\mathrm{Factores}$ & $\mathrm{Externos}$ & & & & \\
\hline Apoyo del gobierno & $\mathrm{M}$ & $\mathrm{M}$ & $\mathrm{M}$ & $\mathrm{B}$ & $\mathrm{A}$ & $\mathrm{M}$ \\
\hline Políticas gubernamentales & $\mathrm{A}$ & $\mathrm{A}$ & $\mathrm{M}$ & $\mathrm{M}$ & $\mathrm{M}$ & $\mathrm{A}$ \\
\hline $\begin{array}{l}\text { Apoyo y servicio de los vendedores } \\
\text { Actitud de la competencia hacia las TI }\end{array}$ & $\mathrm{P}$ & $\mathrm{P}$ & $\mathrm{P}+\mathrm{N}$ & $\mathrm{N}$ & $\mathrm{N}$ & $\mathrm{N}$ \\
\hline Presión por competir & $\mathrm{M}$ & $\mathrm{M}$ & $\mathrm{M}$ & $\mathrm{M}$ & $\mathrm{A}$ & $\mathrm{M}$ \\
\hline
\end{tabular}

Tabla 5 - Resultados de la codificación de entrevistas

A partir de la información contenida en la Tabla 6, se puede ver que la percepción, actitud, liderazgo, estrategia, conocimientos y experiencia son aspectos del factor administrador que inciden directamente sobre la adopción de TI. Por lo tanto, las percepciones del administrador son un factor de peso para la adopción de estas tecnologías en la zona metropolitana de Zacatecas.

En cinco de las empresas estudiadas los empleados son usuarios también de las TI. Sobre este punto se muestra que en las empresas 1 a 5, en general, los empleados tienen buena predisposición hacia las TI, y apoyan las iniciativas de adopción. Las razones de esto son que los administradores contratan a los usuarios ya capacitados o son personas a las que se les da la capacitación específica que el administrador necesita.

El último de los factores internos es el relacionado con los recursos financieros einversión. En las empresas estudiadas se encontró que el administrador evalúa primero los costos y toma esto como el criterio predominante para la adopción de estas tecnologías, además de que ninguna de las empresas estudiadas destina un monto específico a la actualización, adquisición y mantenimiento de TI, sino que conforme se va presentando la necesidad se evalúa la inversión.

Por otro lado, se puede observar que todas las empresas cuentan con la infraestructura de TI suficiente como para dar soporte a los procesos de negocio actuales. Sin embargo, 
esta infraestructura no siempre es compatible al 100\% y está formada más bien por aplicaciones independientes las unas de las otras.

Se puede observar que la mayoría de las empresas estudiadas no perciben presión por parte de los clientes para adoptar TI. Es decir, los clientes atendidos por estas empresas estudiadas, no tienen el nivel de sofisticación necesario, el interés y el conocimiento suficiente en términos de TI como para presionarlas a que adopten estas tecnologías. Ahora bien, el que las empresas estudiadas no tengan una clientela demandante en términos de TI, no implica necesariamente que no consideren incluir alguna estrategia de servicio al cliente apoyada por TI. De la misma manera, las empresas estudiadas consideran utilizar los beneficios de las TI para mejorar su presencia en el mercado, aunque la presión que sienten en este sentido no es alta en este momento.

En las empresas estudiadas se encontró que las políticas gubernamentales nacionales, especialmentelas relacionadas con la facturación electrónica y la contabilidad electrónica, sí han sido factor que estimula la adopción. Algunas de las empresas estudiadas inclusive ven estas disposiciones como una forma de obtener ventajas competitivas o, mínimamente, como una forma de ahorrar. Este factor funciona entonces como un estimulante para la adopción, ya que las empresas que no adopten un conjunto básico de infraestructura de TI, corren el riesgo de no poder seguir operando en la formalidad. A pesar de esto, la percepción general es que el gobierno funciona para ejercer presión, pero no para ofrecer apoyos en capacitación o en simplificación de sus procesos fiscales.

Los vendedores y consultores que sirven a las micro y pequeñas empresas son un factor de adopción importante porque funcionan como la fuente de conocimiento en el área para los administradores. Sin embargo, este tipo de relaciones puede implicar dependencia de las empresas en los productos vendidos. En las empresas estudiadas, esta dependencia se empieza a ver en aquéllas que han adoptado aplicaciones a la medida. Por otro lado, el administrador es quien decide cuándo la tecnología se ha vuelto obsoleta y qué otros usos le puede dar, aparte del que originó su compra. En general, los administradores optan por no cambiar de TI, puesto que ya saben cómo utilizar lo que ya tienen y ya se adaptaron a ella.

Con respecto a la percepción sobre el uso que hace la competencia de las empresas de las TI se encontró que hay administradores que ven que su competencia hace uso intensivo de TI, ante todo en cuestiones de comercio electrónico, pero por otro lado, hay otros administradores que desconocen lo que la competencia está haciendo con las TI. A pesar de esto, la mayoría de los administradores entrevistados sí ven ventajas competitivas en la adopción de TI.

Por último, relacionado con la presión por adoptar TI como lo hace la competencia, las opiniones de los administradores reflejan un interés regular, puesto que consideran que sus ventajas competitivas son, de alguna manera, superiores a las de su competencia. Esto se debe, en parte, a la percepción de los administradores de que son los líderes en el área en la que se desenvuelven.

\section{Conclusiones}

La investigación presentada examina, con un enfoque cualitativo, los factores que afectan la adopción de TI en micro y pequeñas empresas en la zona metropolitana de 
Zacatecas, un área geográfica de bajo desarrollo económico como otros en México y Latinoamérica. La literatura revisada establece que existen factores internos y factores externos que afectan la adopción de TI. Los factores internos se refieren al administrador y sus características, los recursos con los que cuenta la empresa y, por último, los usuarios de las TI. Los factores externos incluyen al gobierno, en forma de las políticas y regulaciones que afectan a las micro y pequeñas empresas, los clientes, la competencia y los vendedores y consultores de TI. Los resultados obtenidos en la investigación, revelan que en las empresas seleccionadas están presentes los factores internos, pero que no ocurre lo mismo con los factores externos. Esto se puede deber a los diferentes contextos que viven las micro y pequeñas empresas seleccionadas para este estudio y las micro y pequeñas empresas seleccionadas en la literatura revisada. Con respecto a los factores internos, un administrador con conocimientos y buenas experiencias en el uso y beneficios de las TI estará dispuesto a disponer de los recursos financieros a su alcance para adoptarlas, puesto que está familiarizado con ellas. Además, un usuario preparado y consciente de los beneficios de estas tecnologías, promoverá iniciativas de adopción. En cambio, un administrador con poca experiencia será renuente a adoptar e invertir en TI y un usuario poco preparado considerará como adecuados los pocos servicios que obtenga de éstas, y por lo tanto, no promoverá iniciativas de adopción.

Sin embargo, con respecto a los factores externos, los clientes de las empresas seleccionadas no tienen la suficiente sofisticación como para ser considerados un factor de peso para la adopción de TI en micro y pequeñas empresas. Asimismo, los administradores entrevistados consideran que las actitudes y percepciones de sus competidores hacia las TI no son muy importantes para la toma de decisiones con respecto a la adopción de TI. Por esto, el factor de la actitud de la competencia hacia la TI y la presión por mantenerse en competencia no son factores de peso para la adopción de TI para las empresas seleccionadas en el estudio. Por otro lado, el gobierno y sus políticas sí son un factor que afecta la adopción, porque las micro y pequeñas empresas estudiadas están más alertas y dispuestas a la adopción, para poder atender dichas disposiciones legales. Además, en las micro y pequeñas empresas estudiadas, el apoyo y servicio postventa de los vendedores de TI es inexistente con respecto a la infraestructura básica. Sin embargo, cuando la venta es de aplicaciones hechas a la medida o de personalización de aplicaciones comerciales que se mantienen en uso, existe una relación más continua entre el vendedor de TI y las micro y pequeñas empresas estudiadas. Por ello, este factor afecta la adopción, en las empresas estudiadas, en tanto los vendedores y consultores estén al pendiente de las necesidades de las empresas a las que proveen de TI y sean capaces de identificar oportunidades de mejora en infraestructura de TI o de apoyar nuevos procesos de negocio en las micro y pequeñas empresas que son ya sus clientes.

La principal limitante de esta investigación se encuentra en su naturaleza cualitativa, por lo que los resultados obtenidos no pueden ser generalizados a todas las micro y pequeñas empresas de la región. Para ello, es necesario un estudio cuantitativo con el que se pueda establecer el impacto de cada factor relacionándolo con los determinantes de intención y uso del modelo de la Teoría Unificada de Aceptación y Uso de Tecnología, UTAUT por sus siglas en inglés (Venkatesh, Thong, \& Xu, 2012).

Finalmente se debe recordar que esta investigación está basada en las percepciones de los administradores de las empresas estudiadas sobre las TI, por lo que también 
se hace necesaria la investigación del lado de la clientela y de los proveedores de infraestructura de TI.

\section{Referencias}

Alam, S. S., \& Noor, M. K. M. (2009). ICT Adoption in Small and Medium Enterprises: an Empirical Evidence of Service Sectors in Malaysia. International Journal of Business and Management, 4(2), 112.

AMIPCI. (2010). Sobre hábitos de los Usuarios de Internet en México Introducción (Vol. 2010). Monterrey, N.L. Retrieved from http://www.amipci.org.mx/temp/ Estudiofinalversion1110-0198933001274287495OB.pdf

Amorós, J. E., Planellas, M., \& Batista-Foguet, J. M. (2007). Does Internet Technology Improve Performance in Small and Medium Enterprises? Evidence From Selected Mexican Firms. Academia Revista Lationamericana de Administración, 39, 71-91.

Antonelli, R. A., Brito-de Almeida, L., Bortolocci-Espejo, M. M. S., \& Longhi, F. L. (2013). Business Professionals' Perceptions Related to the Influence of Information Technology in Individual Work. Journal of Information Systems and Technology Management, 10(1) 41-60. https://dx.doi.org/10.1590/S180717752013000100004.

Aregional. (2010). Reporte nacional sobre la situación competitiva de las MiPyMEs en México, 2010.

Arellano-González, A., Carballo-Mendivil, B., Orrantia-López, M., \& Salazar-Rivera, R. (2013). Diagnóstico de la Madurez de los Procesos de la Cadena de Valor de una Pequeña Empresa Mexicana de Productos de Maíz. Pensamiento Y Gestión, 34, $122-136$.

Arroyo, P. E., Ramírez, J. A., \& Erosa-Martín, V. E. (2007). The Elaboration of a Model to Explain the Adoption of Information Technologies for Supply Chain. In Portland International Center for Management of Engineering and Technology (pp. 2379-2390). Portland, Oregon.

Babbie, E. R. (2009). The Practice of Social Research (12 edition). Boston, MA: Cengage Learning.

Benitez-Amado, J., Llorens-Montes, F. J., \& Perez-Arostegui, M. N. (2010). Information Tecnhology-Enabled Intrapreneurship Culture and Firm Performance. Industrial Management \& Data Systems, 110(4), 550-566.

Biasca, R. E. (2010). ¿Puede Competir la PyME Latinoamericana en el Mundo Actual. Revista Sotavento, (13), 8-23.

Botelho, A. J., \& Bastos-Tigre, P. (2005). Information and Communication Technology (ICT) for Development of Small and Medium-Sized Exportes in Latin America: Brazil. Retrieved from http://www.cepal.org/en/publications/3699-informationand-communication-technology-ict-development-small-and-medium-sized. 
Brewerton, F. J., \& Feinberg, M. (2011). Perceptions of Internet Technology in Small Businesses: An Empirical Comparison of The U.S., México and Croatia. Review of Business Information Systems, 8(3), 29-38.

Bruque, S., \& Moyano, J. (2007). Organisational Determinants of Information Technology Adoption and Implementation in SMEs: The Case of Familiy and Cooperative Firms. Technovation, 27(5), 241-253.

Búrca, S., Fynes, B., \& Marshall, D. (2005). Strategic Technology Adoption: Extending ERP Across the Supply Chain. Journal of Enterprise Information Management, 18(1), 427-440.

Chan, S. C. H., \& Ngai, E. W. T. (2007). A Qualitative Study of Informaton Technology Adoption: How Ten Organizations Adopted Web-Based Training. Information Systems Journal, $17(3), 289-315$.

Chao, C., \& Chandra, A. (2012). Impact of Owner's Knowledge of Information Technology on Strategic Alignment and IT Adoption in US Small Firms. Journal of Small Business and Enterprise Development, 19(1), 114-131.

Chesney, T. (2010). Competitive Information in Small Businesses. Norwell, MA.: Kluwer Academic Publishers.

Cragg, P., King, M., \& Hussin, H. (2002). IT Alignment and Firm Performance in Small Manufacturing Firms. The Journal of Strategic Information Systems, 11(2), 109132.

de León-Sigg, M., Villa-Cisneros, J. L., Vazquez-Reyes, S., \& Rentería-Salcedo, J. A. (2013). Aplicación del Modelo del Usuario Perezoso para Explicar la Adopción de Tecnologías de Información en Pequeñas Empresas. In Proceedings of the 2nd International Conference on Software Process Improvement. Zacatecas, Zac.: CIMAT, Unidad Zacatecas.

Drew, S. (2003). Strategic Uses of E-Commerce by SMEs in the East of England. European Management Journal, 21(1), 79-88.

Erosa-Martín, V. E., \& Arroyo-López, P. E. (2010). Segmentation of Small Firms Based on Information Technology Usage and Absorptive Capabilities. In Proceedings of the Technology Management for Global Economic Growth (PICMET), 2010 (p. 8). Phuket, Thailand.

Fathian, M., Akhavan, P., \& Hoorali, M. (2008). E-readiness Assessment of Non-Profit ICT SMEs in a Developing Country: The Case of Iran. Technovation, 28(9), 578-590.

Fink, D., \& Disterer, G. (2006). International Case Studies: To What Extent is ICT Infused into the Operations of SMEs? Journal of Enterprise Information Management, 19(6), 608-624.

Ghobakhloo, M., Arias-Aranda, D., \& Benitez-Amado, J. (2011). Information Technology Implementation Success Within SMEs in Developing Countries: An Interactive Model. In Proceedings of the POMS 22nd Annual Conference: Operations Management: The Enabling Link. Reno, E. U. 
Ghobakhloo, M., Hong, T. S., Sabouri, M. S., \&Zulkifli, N. (2012). Strategies for Successful Information Technology Adoption in Small and Medium-sized Enterprises. Information, 3(4), 36-67. http://doi.org/10.3390/info3010036

González-Peyro, I. A., Torres-Nájera, J. J., \& Sifuentes-García, G. M. (2012). Uso e Implementación de las Tecnologías de Información y Comunicación en la Toma de Decisiones en los Hoteles de la Ciudad de Durango. Revista de La Alta Tecnología Y La Sociedad, 6(1), 1-15.

Guerrero-Cuéllar, R., \& Rivas-Tovar, L. A. (2005). Comercio Electrónico en México: Propuesta de un Modelo Conceptual Aplicado a las PyMEs. Revista Internacional de Ciencias Sociales Y Humanidades SOCIOTAM, 15(1), 79-116.

Hernández Sampieri, R. (2010). Metodología de la investigación (5ta Edició). McGrawHill Interamericana.

Ihlström, C., Magnusson, M., Scupola, A., \& Tuunainen, V. K. (2003). SME Barriers to Electronic Commerce Adoption: Nothing Changes, Everything is New. In G. Gingrich (Ed.), Managing IT in Government, Business \& Communities (pp. 147163). Hershey, PA: Idea Group Publishing.

INEGI. (2013). Clasificación de Municipios de Zacatecas. Retrieved August 15, 2013, from http://sc.inegi.gob.mx/niveles/index. jsp?me $=$ na\&ly $=00,32 \& l a=32 \& t 2=$ ZACATECAS\&at $=\& n e=e s \& n t=25 \#$

Jabar, M. A., Sidi, F., Selamat, M., Ghani, A., \& Ibrahim, H. (2009). An Investigation into Methods and Concepts of Qualitative Research in Information System Research. Computer and Information Science, 2(4), 47-54.

Jones, C., Motta, J., \& Alderete, M. V. (2016). Gestión Estratégica de Tecnologías de Información y Comunicación y Adopción del Comercio Electrónico en Mipymes de Córdoba, Argentina. Estudios Gerenciales, 32(138).

Kuwayama, M. (2001). E-Commerce and Export Promotion Policies for Small and Medium Sized Enterprises: East Asian and Latin American Experiences. Santiago, Chile.

Love, P., Irani, Z., Standing, C., Lin, C., \& Burn, J. M. (2005). The Enigma of Evaluation: Benefits, Costs and Risks of IT Australian Small-Medium-Sized Enterprises. Information \& Management, 42(7), 947-964.

Maldonado-Guzmán, G., Martínez-Serna, M. del C., García Perez de Lema, D., Aguilera Enríquez, L., \& González Adame, M. (2010). La Influencia de las TICs en el Rendimiento de la PyME de Aguascalientes. Investigación Y Ciencia de La Universidad Autónoma de Aguascalientes, 47, 57-65.

Merritt, H. (2012). Factors Affecting the Adoption of an Emerging Technology: The Diffusion of Wi-Fi Internet in México. In Proceedings of the Technology Management for Emerging Technologies (PICMET 2012) (pp. 1440-1449). Vancouver, BC: IEEE Xplore.

Middleton, K. L., \& Byus, K. (2011). Information and Communications Technology Adoption and Use in Small and Medium Businesses: The Influence of Hispanic Ethnicity. Management Research Review, 34(1), 98-110. 
Mole, K., \& Ghobadian, A. (2004). The Use and Deployment of Soft Process Technologies within UK Manufacturing SMEs: An Empirical Assessment Using Logit Models. Journal of Small Business Management, 42(3), 303-324.

Myers, M. D., \& Baskerville, R. (2004). Special Issue on Action Research in Information Systems: Making IS Research Relevant to Practice. MIS Quaterly, 28(3), 329-335.

Neuendorf, K. (2001). The Content Analysis Guidebook. Thousand Oaks, CA: SAGE Publications.

Nguyen, T. H. (2009). Information Technology Adoption in SMEs: An Integrated Framework. International Journal of Entrepreneurial Behavior and Research, 15(2), 162-186.

Nguyen, T. H., Newby, M., \& Macaulay, M. J. (2013). Information Technology Adoption in Small Business: Confirmation of a Proposed Framework. Journal of Small Business Management, n/a-n/a. http://doi.org/10.1111/jsbm.12058

Novaes-Zilber, S., \& Braz-de Araújo, J. (2012). Small Companies Innovations in Emerging Countries: E-Business Adoption and its Business Model. Journal of Technology Management and Innovation, 7(2), 102-116.

O’Mahony, D., Wright, G., Yogeswaran, P., \& Govere, F. (2014). Knowledge and Attitudes of Nurses in Community Health Centres about Electronic Medical Records. Curationis, 37(1), 6.

Parida, V., Johansson, J., Ylinenpää, H., \& Baunerhjelm, P. (2010). Barriers to information and communication technology adoption in small firms. Retrieved from http://entreprenorskapsforum.se/swe/wp-content/uploads/2010/o9/WP_ Barriers_to_ICT_adoption.pdf

Pontikakis, D., Lin, Y., \& Dermibas, D. (2006). History Matters in Greece: The Adoption of Internet-Enabled Computers by Small and Medium Sized Enterprises. Information Economics and Policy, 18(3), 332-358.

Premkumar, G. (2003). A Meta-Analysis of Research on Information Technology Implementation in Small Business. Journal of Organizational Computing and Electronic Commerce, 13(2), 91-121.

Qureshi, S., Kamal, M., \& Wolcott, P. (2009). Information Technology Interventions for Growth and Competitiveness in Micro-Enterprises. International Journal of E-Business Research (IJEBR), 5(1), 117-140.

Qureshil, S., \& York, A. S. (2008). Information Technologoy Adoption by Small Businesses in Minority and Ethnic Communities. In Proceedings of the 41st Annual Hawaii International Conference on System Sciences. IEEE.

Ramayah, T., Ling, N. S., Taghizadeh, S. K., \& Rahman, S. A. (2015). Factors Influencing SMEs Website Continuance Intention in Malaysia. Telematics and Informatics, 33(2016), 150-164.

Ramdani, B., Kawalek, P., \& Lorenzo, O. (2009). Predicting SMEs'Adoption of Enterprise Systems. Journal of Enterprise Information Management, 22(1/2), 10-24. 
Rese, A., Schreiber, S., \& Baier, D. (2014). Technology Acceptance Modeling of Augmented Reality at the Point of Sale: Can Surveys be Replaced by an Analysis of Online Reviews? Journal of Retailing and Consumer Services, March. http://doi. org/http://dx.doi.org/10.1016/j.jretconser.2014.02.011

Riemenschneider, C., Harrison, D. A., \& Mykytyn, P. P. (2003). Understanding IT adoption Decisions in Small Business: Integrating Current Theories. Information \& Management, 40(4), 269-285.

Rubio Bañón, A., \& Aragón Sánchez, A. (2002). Factores Explicativos del Éxito Competitivo. Un Estudio Empírico en la PyME. Cuadernos de Gestión, 2(1), 49-63.

Sá, F., \& Rocha, Á. (2012). Definição da arquitetura de informação em organismo da administração pública local. RISTI - Revista Ibérica de Sistemas e Tecnologias de Informação, (10), 51-64.

Salmeron, J. L., \& Bueno, S. (2006). An Information Technologies and Information Systems Industry-based Classification in Small and Medium-Sized Enterprises : An institutional view. European Journal of Operational Research, 173(3), 1012-1025. http://doi.org/10.1016/j.ejor.2005.07.002

Sarosa, S., \& Zowghi, D. (2003). A Strategy for Adopting Information Technology for SMEs : Experience in Adopting Email within an Indonesian Furniture Company. Electronic Journal of Information Systems Evaluation, 6(2), 165-176.

Secretaría de Desarrollo Social, Consejo Nacional de Población, \& INEGI. (2004). Delimitación de las Zonas Metropolitanas de México. Retrieved from http://www. conapo.gob.mx/en/CONAPO/Delimitacion_de_las_zonas_metropolitanas_de_ Mexico.

Secretaría de Gobernación. (2009). Acuerdo por el que se establece la estratificación de las micro, pequeñas y medianas empresas. Diario Oficial de La Federación. México, D.F. Retrieved from http://dof.gob.mx/nota_detalle. php?codigo $=5096849 \&$ fecha $=30 / 06 / 2009 \&$ print $=$ true

Seyal, A. H., Rahim, M. M., \& Rahman, M. N. (2000). An Empirical Investigation of Use of Information Technology among Small and Medium Business Organizations: A Bruneian Scenario. The Electronic Journal of Information Systems in Developing Countries, 2, 1-17.

Siqueira, A. C., \& Fleury, M. T. L. (2011). Complementarities of Human Capital and Information Technology: Small Businesses, Emerging Economy Context and the Strategic Role of Firm Resources. Technology Analysis and Strategic Management, 23(6), 693-653.

Spencer, A., Buhalls, D., \& Moital, M. (2012). A Hierarchical Model of Technology Adoption for Small Owner-Managed Travel Firms: An Organizational DecisionMaking and Leadership Perspective. Tourism Management, 33(5), 1195-1208.

Stockdale, R., \& Standing, C. (2004). Benefits and Barriers of Electronic Marketplace Participation: an SME perspective. Journal of Enterprise Information Management, 17(4), 301-311. 
Tan, K. S., Chong, S. C., Lin, B., \& Eze, U. C. (2009). Internet-Based ICT Adoption: Evidence from Malaysian SMEs. Industrial Management \& Data Systems, 109(2), 224-244.

Thong, J. Y. L. (2001). Resource Constraints and Information Systems Implementation in Singaporean Small Businesses. Omega, 29(2), 143-156.

Trejo Nieto, A. (2013). Las Economías de las Zonas Metropolitanas de México en los Albores del Siglo XXI. Estudios Demográficos Y Urbanos, 28(3), 545-591.

Ueki, Y., Tsuji, M., \& Cárcamo-Olmos, R. (2005). Tecnología de la Información y las Comunicaciones ( TIC ) para el Fomento de las PyMEs Exportadoras en América Latina y Asia Oriental. Retrieved from http://archivo.cepal.org/pdfs/2005/ S2005047.pdf

Utomo, H., \& Dodgson, M. (2001). Contributing Factors to the Diffusion of IT within Small and Medium-Sized Firms in Indonesia. Journal of Global Information Technology Management, 4(2), 22-37.

Venkatesh, V., Thong, J. Y. L., \& Xu, X. (2012). Consumer Acceptance and Use of Information Technology: Extending the Unified Theory of Acceptance And Use of Technology. MIS Quaterly, 36(1), 157-178.

Walczuch, R., Van Braven, G., \& Lundgren, H. (2000). Internet Adoption Barriers for Small Firms in the Netherlands. European Management Journal, 18(5), 561-572.

Walsham, G. (2006). Doing interpretive research. European Journal of Information Systems, 15(3), 320-330. http://doi.org/10.1057/palgrave.ejis.3000589

Ward, J. M., \& Caldeira, M. M. (2003). Using Resource-Based Theory to Interpret the Successful Adoption and Use of Information Systems and Technology in Manufacturing Small and Medium-Sized Enterprises. European Journal of Information Systems, 12(2), 127-141.

Yen, P. Y., \& Bakken, S. (2012). Review of Health Information Technology Usability Study Methodologies. Journal of the American Medical Informatics Association, 19(3), 413-422. 